
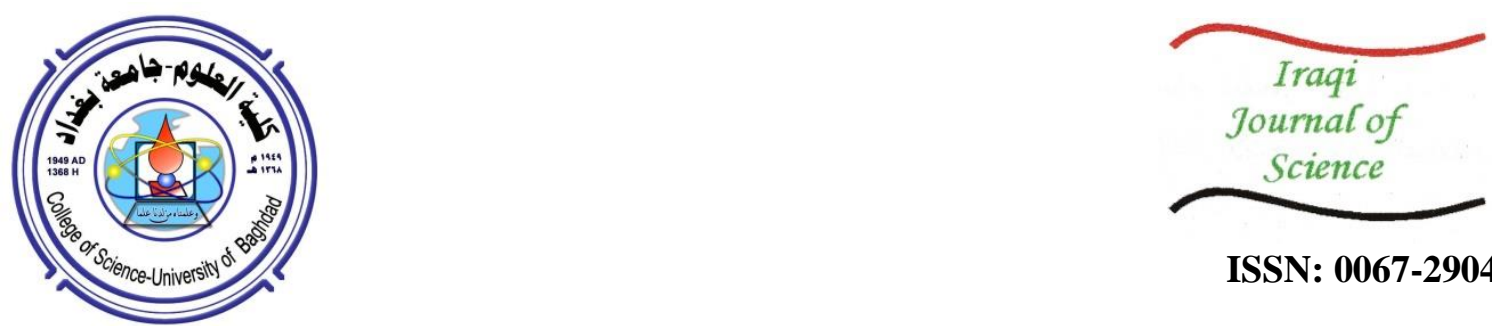

ISSN: 0067-2904

\title{
Study of the Nuclear Structure for Some Target Nuclei Used in the Production of Beta-Emitting Radioactive Isotopes for the Fabrication of Nuclear Batteries
}

\author{
Ali A. Alzubadi* and Samaa S. Nassir \\ Department of Physics, College of Science, University of Baghdad, Baghdad, Iraq
}

Received: 23/11/2019 Accepted: 30/4/2020

\begin{abstract}
The nuclear structure of ${ }^{40} \mathrm{Ar},{ }^{112} \mathrm{Cd},{ }^{133} \mathrm{Cs},{ }^{151} \mathrm{Eu},{ }^{154} \mathrm{Sm}$, and ${ }^{226} \mathrm{Ra}$ target nuclei used in nuclear battery technology was investigated. These nuclei are widely used for the radioisotope thermo-electric generator space studies and for betavoltaic battery microelectronic systems. For this purpose, some nuclear static properties were calculated. In particular, the single particle radial nuclear density distribution, the corresponding root mean square radii, neutron skin thicknesses, and binding energies were calculated within the framework of Hartree-Fock approximation with Skyrme interaction. The bremsstrahlung spectra produced by the absorption of beta particles through the betavoltaic process and backscattered photons spectrum were also studied. All the obtained results were compared with available experimental data.
\end{abstract}

Keywords: Hartree-Fock, Nuclear Batteries, Betavoltaic, Bremsstrahlung Emission.

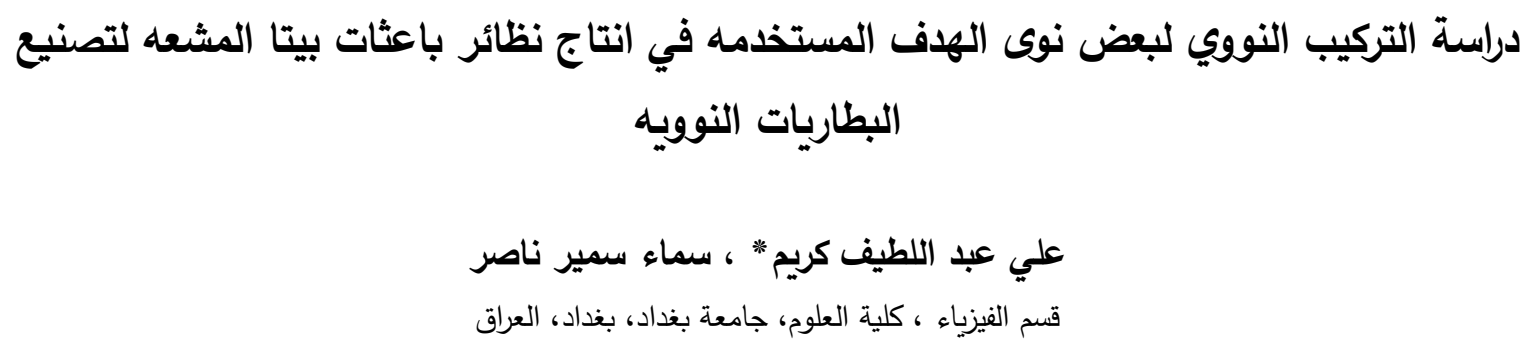

$$
\begin{aligned}
& \text { الخلاصه } \\
& \text { تم دراسة التركيب النووي لأنوية الهدف }{ }^{226} \mathrm{Ra} \text { و ل }
\end{aligned}
$$

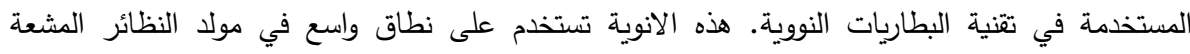

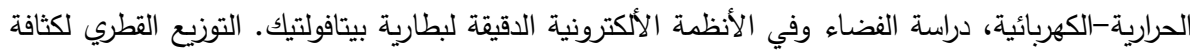

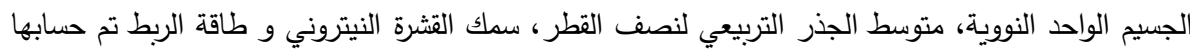

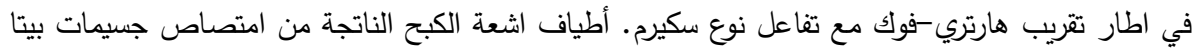

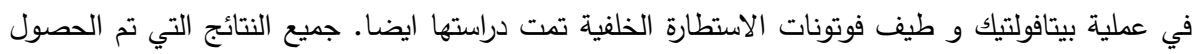

$$
\begin{aligned}
& \text { عليها تمت مقارنتها مع النتائج العملية المتوفرة. }
\end{aligned}
$$

\section{Introduction}

Because" of their. growing use as a . "long-life" "source" of energy, nuclear batteries have a significant position in technology, particularly in "space-craft" and microelectronic 
applications. Nuclear batteries convert thermal energy resulting from the decay of radioactive material into .electricity [1,2]. There are two main types of nuclear batteries, which are the thermal converting and non-thermal converting such as betavoltaics [3]. This type of batteries converts energy disintegration into electricity via $p$-n junctions using semiconductor devices, because of the greater densities of energy and the longer radioisotope life relative to the other types [4]. "Betavoltaics" are used as power suppliers in "medical and "microelectronic applications" [5].

The nuclear static properties of some target nuclei used for the production of radioactive nuclei which are employed in nuclear batteries, namely ${ }^{40} \mathrm{Ar},{ }^{112} \mathrm{Cd},{ }^{133} \mathrm{Cs},{ }^{151} \mathrm{Eu},{ }^{154} \mathrm{Sm}$, and ${ }^{226} \mathrm{Ra}$, have been calculated in the framework of Hartree-Fock (HF) approximation with Skyrme type interaction. "In particular, "“charge", "proton, mass," and "neutron densities," as well as their "associated values of root mean square" (rms) "radii", nuclear binding "energy," and neutron skin. Thickness, have been calculated. Besides, the simulation of bremsstrahlung emission by beta particles, using numerical cross-section and backscattered photons, was applied.

\section{Theoretical Framework}

The microscopic mean-field (MF) methods have been widely used in studying the "ground" state nuclear properties of stable as well as unstable nuclei. In this context, the two-body effective interaction models of Skyrme [6] and Gogny [7] are the most commonly used with the non-relativistic HF method. The "analytic" simplicity of the Skyrme interaction allows for the definition of model parameters, which "integrate" basic nuclear properties [8].

In the present work, the $\mathrm{SkM}^{*}$ parameterization, with its numerical values listed in Table-1 [9], were implemented with the HF method. This parameterization in the actinide region was adapted to fission obstacles and surface tension. SkM* is an expanded version of SkM for large deformations and could be used for astrophysical purposes such as the calculations of neutron stars [10].

Table 1-Skyrme parameterization used in the present work

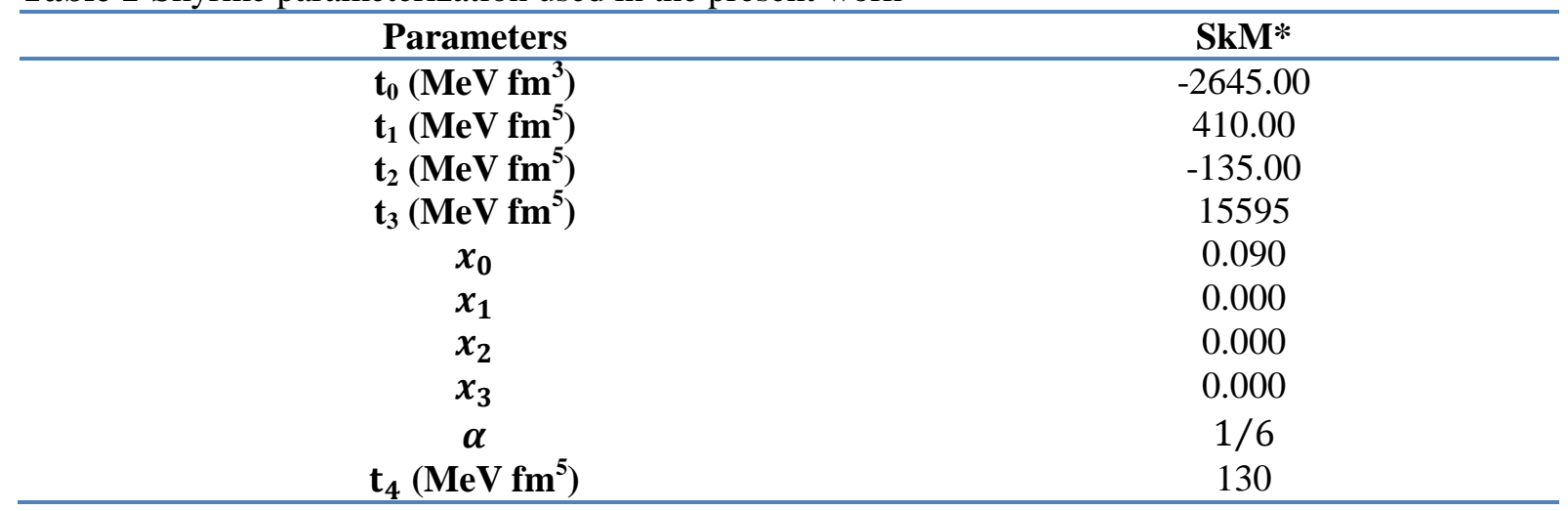

The Skyrme Hartree-Fock (SHF) equation is derived from the variation principle of the ground state of "wave function of the nucleus", which is assumed as a "Slater determinant" of the single particle state $\varphi_{\beta}$

$$
\varphi_{\beta}\left(x_{1}, x_{2}, \ldots, x_{A}\right)=\frac{1}{\sqrt{A !}} \operatorname{det}\left|\varphi_{\beta}\left(x_{j}\right)\right|
$$

where $x$ denotes the set of space $(\vec{r})$, spin $(\sigma)$, and isospin coordinate $(q)$. The expected value for the total Hamiltonian $(H)$ of such Slater determinant can be written as the integral of the Hamiltonian density $(H)$

$$
\langle\varphi|H| \varphi\rangle=\int H(\vec{r}) d \vec{r}
$$

For the Skyrme interaction, the Hamiltonian density is given as a function of the "nucleon density" $\left(\rho_{q}\right)$, "kinetic energy" density" $\left(\tau_{q}\right)$, and spin orbit density $\left(\vec{J}_{q}\right)$, where $q$ stands for "proton or neutron". It can be written as the a sum of terms associated with different parts of the force [11]

$$
H=H_{\text {kin }}+H_{0}+H_{3}+H_{\text {eff }}+H_{\text {fin }}+H_{\text {so }}+H_{\text {sg }}+H_{\text {Coul }}
$$

where $H_{\text {kin }}$ is the kinetic energy term, $H_{0}$ is the zero range term, $H_{3}$ is density dependent term, $H_{e f f}$ is the effected mass term, $H_{\text {fin }}$ is the finite range term, $H_{s o}$ is the spin-orbit term, $H_{s g}$ is the tensor coupling with spin and gradient term, and $H_{\text {Coul }}$ is the Coulomb term. The terms in Eq. 3 are defined as follows: 


$$
\begin{aligned}
& H_{\text {kin }}=\frac{\hbar^{2}}{2 m} \tau \\
& H_{0}=\frac{t_{0}}{2}\left(1+\frac{x_{0}}{2}\right) \rho^{2}-\frac{t_{0}}{2}\left(x_{0}+\frac{1}{2}\right) \sum_{q} \rho_{q}^{2} \\
& H_{3}=\frac{t_{3}}{12}\left(1+\frac{x_{3}}{2}\right) \rho^{2+\alpha}-\frac{t_{3}}{12}\left(x_{3}+\frac{1}{2}\right) \rho^{\alpha} \sum_{q} \rho_{q}^{2} \\
& H_{\text {eff }}=\frac{1}{4}\left(t_{1}\left(1+\frac{x_{1}}{2}\right)+t_{2}\left(1+\frac{x_{2}}{2}\right)\right) \rho \tau-\frac{1}{4}\left(t_{1}\left(x_{1}+\frac{1}{2}\right)-t_{2}\left(x_{2}+\frac{1}{2}\right)\right) \sum_{q} \rho_{q} \tau_{q} \\
& \boldsymbol{H}_{\text {fin }}=\frac{\mathbf{1}}{\mathbf{1 6}}\left(\mathbf{3} \boldsymbol{t}_{\mathbf{1}}\left(\mathbf{1}+\frac{\boldsymbol{x}_{\mathbf{1}}}{\mathbf{2}}\right)-\boldsymbol{t}_{\mathbf{2}}\left(\mathbf{1}+\frac{\boldsymbol{x}_{\mathbf{2}}}{\mathbf{2}}\right)\right)(\boldsymbol{\nabla} \boldsymbol{\rho})^{2}-\frac{\mathbf{1}}{\mathbf{1 6}}\left(\boldsymbol{t}_{\mathbf{1}}\left(\boldsymbol{x}_{\mathbf{1}}+\frac{\mathbf{1}}{\mathbf{2}}\right)+\boldsymbol{t}_{\mathbf{2}}\left(\boldsymbol{x}_{\mathbf{2}}+\frac{\mathbf{1}}{\mathbf{2}}\right)\right) \sum_{\boldsymbol{q}}\left(\boldsymbol{\nabla} \boldsymbol{\rho}_{\boldsymbol{q}}\right)^{\mathbf{2}} \\
& H_{\text {so }}=\frac{t_{4}}{2}\left(\vec{J} \cdot \nabla \rho+\sum_{q} \vec{J}_{q} \cdot \nabla \rho_{q}\right) \\
& H_{\text {sg }}=-\frac{1}{16}\left(t_{1} x_{1}+t_{2} x_{2}\right) \vec{J}^{2}+\frac{1}{16}\left(t_{1}-t_{2}\right) \sum_{q} \vec{J}_{q}^{2} \\
& H_{\text {Coul }}=\frac{e^{2}}{2} \rho_{p}(\vec{r}) \int \frac{\rho_{p}\left(\vec{r}^{\prime}\right)}{\left|\vec{r}-\vec{r}^{\prime}\right|} \overrightarrow{d r}^{\prime}-\frac{3 e^{2}}{4}\left(\frac{3}{\pi}\right)^{1 / 3} \rho_{p}^{4 / 3}(\vec{r})
\end{aligned}
$$

where $H_{\text {Coul }}$ consists of the direct and exchange contributions and $t_{0}, t_{1}, t_{2}, t_{3}, x_{0}, x_{1}, x_{2}, x_{3}, \alpha$ and $t_{4}$ are the Skyrme parameterizations which are usually determined by fitting the experimental ground state properties of the spherical nuclei. $\rho_{q}, \tau_{q}$ and $J_{q}$ are the densities distributions, "kinetic energy" density, and spin orbit density for the nucleon, respectively. The non-indexed quantities represent the total density:

$\rho=\rho_{p}+\rho_{n} ; \quad \tau=\tau_{p}+\tau_{n} ; \quad \vec{J}=\overrightarrow{J_{p}}+\overrightarrow{J_{n}}$. These densities are calculated from the single particle which waves the function $\varphi_{\beta}$ defining the Slater determinant:

$$
\begin{aligned}
& \rho_{q}(\vec{r})=\sum_{\sigma, \beta}\left|\varphi_{\beta}(\vec{r}, \sigma, q)\right|^{2}, \\
& \tau_{q}(\vec{r})=\sum_{\sigma, \beta}\left|\nabla \varphi_{\beta}(\vec{r}, \sigma, q)\right|^{2}, \\
& \overrightarrow{J_{q}}(\vec{r})=(-i) \sum_{\sigma, \beta, \sigma^{\prime}} \varphi_{\beta}^{*}(\vec{r}, \sigma, q)\left[\nabla \varphi_{\beta}(\vec{r}, \grave{\sigma}, q)\right] \times\left\langle\sigma|\vec{\sigma}| \sigma^{\prime}\right\rangle
\end{aligned}
$$

The SHF equation is obtained by writing that the total energy $E$ is stationary with respect to the variation of the single particle state, $\varphi_{\beta}$, with the subsidiary condition that $\varphi_{\beta}$ is normalized

$$
\frac{\delta}{\delta \varphi_{\beta}} E-\sum_{\beta} \in_{\beta} \int\left|\varphi_{\beta}(\vec{r})\right|^{2} d \vec{r}=0
$$

Using the explicit from of the Hamiltonian density in Eq. 3, one can derive the equation for the single particle wave function $\varphi_{\beta}$

$$
\left[-\nabla \frac{\hbar^{2}}{2 m_{q}^{*}(\vec{r})} \nabla+U_{q}(\vec{r})-i \vec{W}_{q}(\vec{r}) \cdot(\nabla \times \sigma)\right] \varphi_{\beta}=\epsilon_{\beta} \varphi_{\beta}
$$

where $q$ is the charge of the single-particle state $\beta$ and the single particle wave function $\varphi_{\beta}$ containing all the quantum effect of the system. Eq. 7 has a form of local Schrödinger equation with an effective mass $m_{q}^{*}(\vec{r})$ that depends on density only [11]

$$
\frac{\hbar^{2}}{2 m_{q}^{*}(\vec{r})}=\frac{\hbar^{2}}{2 m}+\frac{1}{8}\left[t_{1}\left(1+\frac{x_{1}}{2}\right)+t_{2}\left(1+\frac{x_{2}}{2}\right)\right] \rho-\frac{1}{8}\left[t_{1}\left(x_{1}+\frac{1}{2}\right)+t_{2}\left(x_{2}+\frac{1}{2}\right)\right] \rho_{q}
$$

whereas the central potential depends also on kinetic energy density 


$$
\begin{aligned}
U_{q}=t_{0}\left(1+\frac{x_{0}}{2}\right) & \rho-t_{0}\left(x_{0}+\frac{1}{2}\right) \rho_{q} \\
& +\frac{t_{3}}{12} \rho^{\alpha}\left[(2+\alpha)\left(1+\frac{x_{3}}{2}\right) \rho-2\left(\frac{1}{2}+x_{3}\right) \rho_{q}-\alpha\left(\frac{1}{2}+x_{3}\right) \frac{\rho_{p}+\rho_{n}}{\rho}\right] \\
& +\frac{1}{4}\left[t_{1}\left(1+\frac{x_{1}}{2}\right)-t_{2}\left(1+\frac{x_{2}}{2}\right)\right] \tau-\frac{1}{4}\left[t_{1}\left(x_{1}+\frac{1}{2}\right)-t_{2}\left(x_{2}+\frac{1}{2}\right)\right] \tau_{q} \\
& -\frac{1}{8}\left[3 t_{1}\left(1+\frac{x_{1}}{2}\right)-t_{2}\left(1+\frac{x_{2}}{2}\right)\right] \nabla^{2} \rho+\frac{1}{8}\left[3 t_{1}\left(x_{1}+\frac{1}{2}\right)+t_{2}\left(x_{2}+\frac{1}{2}\right)\right] \nabla^{2} \rho_{q} \\
& +\frac{1}{8}\left(t_{1}-t_{2}\right) \vec{J}_{q}-\frac{1}{8}\left(x_{1} t_{1}-x_{2} t_{2}\right) \vec{J}+\delta_{q . p r o t o n} U_{\text {Coul }}
\end{aligned}
$$

The spin-orbit potential is given by

$$
\vec{W}_{q}=\frac{t_{4}}{2}\left(\nabla \rho+\nabla \rho_{q}\right)
$$

and the Coulomb potential, where the exchange part is calculated with Slater approximations which has a form of

\section{Calculation Method}

$$
U_{\text {Coul }}=\frac{e^{2}}{2} \int \frac{\rho_{p}\left(\vec{r}^{\prime}\right)}{\left|\vec{r}-\vec{r}^{\prime}\right|} \overrightarrow{d r}^{\prime}-e^{2}\left(\frac{3}{\pi}\right)^{1 / 3} \rho_{p}^{1 / 3}(\vec{r})
$$

The HF equation Eq. (7) is solved self-consistently to obtain the desired wave function for single-particle state, $\varphi_{\beta}$. After the wave function is obtained, the binding energy of the nucleus is calculated according to Eq. (2). The densities and local potential are then evaluated using Eqs. (5) and (9), respectively. In performing the calculations, we use the computer code for spherical SHF+BCS [12] where the contribution of pairing interaction to the binding energy of the nucleus is calculated as

$$
E_{\text {pair }}=-\sum_{q} G_{q}\left[\sum_{\beta \in q} \sqrt{\omega_{\beta}\left(1-\omega_{\beta}\right)}\right]^{2}
$$

where the pairing matrix elements $\left(G_{q}\right)$ are constant within each species $q \in\{p, n\}$. The occupation probability $\left(\omega_{\beta}\right)$ is determined by solving the Bardeen-Cooper-Schrieffer (BCS) pairing equation

$$
\omega_{\beta}=\frac{1}{2}\left[1-\frac{\epsilon_{\beta}-\epsilon_{F, \beta}}{\sqrt{\left(\epsilon_{\beta}-\epsilon_{F, \beta}\right)^{2}+\Delta_{q}^{2}}}\right]
$$

The pairing gap $\left(\Delta_{q}\right)$ and the Femi energy $\left(\epsilon_{F, \beta}\right)$ are determined by simultaneous solution of the gap equation and the particle number condition

$$
\frac{\Delta_{q}}{G_{q}}=\sum_{\beta \in q} \sqrt{\omega_{\beta}\left(1-\omega_{\beta}\right)} \quad ; \quad A_{q}=\sum_{\beta \in q} \omega_{\beta}
$$

where $A_{q}$ is the desired number of protons and neutrons. In solving Eq.(13), we use a constant gap approach for proton and neutron

where $A$ is a nucleon number.

$$
\Delta_{p}=\Delta_{n}=\frac{11.2}{\sqrt{A}} \mathrm{MeV}
$$

The "rms radii" of proton, charge, neutron, and mass distributions can be evaluated from Eqs. $5 \mathrm{a}, 5 \mathrm{~b}$ and $5 \mathrm{c}$ as [13]:

$$
r_{q}=\left\langle r_{q}^{2}\right\rangle^{1 / 2}=\left[\frac{\int r^{2} \rho_{q}(\vec{r}) d \vec{r}}{\int \rho_{q}(\vec{r}) d \vec{r}}\right]^{1 / 2}
$$

The neutron thickness can be given as [14]:

$$
t_{n}=r_{n}-r_{p}
$$

where $r_{n}$ is the rms of neutron and $r_{p}$ is the rms of proton.

The cross-section $\sigma\left(W_{e}, k\right)$ for the production of "bremsstrahlung" photon of energy between $k$ and $+d k$ is integrated by an electron with total energy $W_{0}$ in all photon directions (including "rest-mass" energy $m_{0} c^{2}$ ) when it interacts with the Coulomb "field" of 
target nucleus of atomic number $\mathrm{Z}$ and atomic mass $\mathrm{A}$. The expression for the crosssection is given by [15-17]:

$$
\begin{gathered}
\sigma\left(W_{e}, k\right)=\frac{Z^{2} r_{\circ}^{2}}{137} \frac{d k}{k} \frac{P}{P_{e}}\left\{\frac{4}{3}-2 W_{e} W \frac{P_{e}^{2}+P^{2}}{P_{e}^{2}+P^{2}}+\frac{\epsilon_{e} W}{P_{e}^{3}}+\frac{\epsilon W_{e}}{P^{3}}-\frac{\epsilon_{e} \epsilon}{P_{\circ} P}+L\left[\frac{8}{3} \frac{W_{e} W}{P_{e} P}+\frac{k^{2}\left(W_{e}^{2} W^{2}+P_{e}^{2} P^{2}\right)}{P_{e}^{3} P^{3}}+\right.\right. \\
\left.\left.\frac{k}{2 P_{e} P}\left(\frac{W_{e} W+P_{e}^{2}}{P_{e}^{3}} \epsilon_{e}-\frac{W_{e} W+P^{2}}{P^{3}} \epsilon+\frac{2 k W_{e} W}{P_{e}^{2} P^{2}}\right)\right]\right\}
\end{gathered}
$$

where $r_{0}$ is the classical electron radius $=e^{2} / m_{o} c^{2}=2.818 \times 10^{-3} \mathrm{~cm}, W_{e}, P_{e}$ and $W, P$ are the initial and final values of electron energy and momentum in units of $m_{0} c^{2}$ and $m_{0} c$, respectively, and $k$ is the photon energy in $m_{o} c^{2}$ units.

The energy of the backscatter peak is given by:

where $E_{b . s}$ is the backscattering energy

$$
E_{b . s}=E_{\gamma}-E_{C}=\frac{E_{\gamma}}{1+\frac{2 E_{\gamma}}{m_{o} c^{2}}}(\mathrm{MeV})
$$

$$
\begin{aligned}
& E_{\gamma} \text { is gamma energy } \\
& E_{C} \text { is Compton energy }
\end{aligned}
$$

\section{Results and Discussion}

The main concern of the present work is to calculate the most common static nuclear properties for some selected target nuclei used for the production of pure beta emitter used in the fabrication of nuclear batteries. The discussion of the results of the nuclear structure will be divided into two parts; the first part is where most features of the nuclear ground state for ${ }^{40} \mathrm{Ar},{ }^{112} \mathrm{Cd},{ }^{133} \mathrm{Cs},{ }^{151} \mathrm{Eu},{ }^{154} \mathrm{Sm}$, and ${ }^{226} \mathrm{Ra}$ nuclei were studied with the aid of the HF method and SkM* Skyrme parameterization. The second part deals with the bremsstrahlung spectrum and backscattered photons produced by the absorption of beta particles emitted from the product nuclei, namely ${ }^{42} \mathrm{Ar},{ }^{113} \mathrm{Cd},{ }^{134} \mathrm{Cs},{ }^{152} \mathrm{Eu},{ }^{155} \mathrm{Eu}$, and ${ }^{227} \mathrm{Ac}$ via $(n, \gamma)$ reaction (Table-2). All obtained results are compared with available experimental data.

Table 2-The target and product nuclei used in the present work

\begin{tabular}{ccccccc}
\hline $\begin{array}{c}\text { Target } \\
\text { nuclei }\end{array}$ & $\mathbf{Z}$ & $\mathbf{N}$ & $\begin{array}{c}\text { Decay energy } \\
(\mathbf{M e V})\end{array}$ & Reaction & $\begin{array}{c}\text { Product } \\
\text { nuclei }\end{array}$ & $\boldsymbol{\beta}_{\text {max }}(\mathbf{M e V})$ \\
\hline${ }^{{ }^{\mathbf{4 0}} \mathbf{A r}}$ & 18 & 22 & 0.600 & $(n, \gamma)$ & ${ }^{42} \mathrm{Ar}$ & 0.6000 \\
${ }^{{ }^{112} \mathbf{C d}}$ & 48 & 64 & 0.580 & $(n, \gamma)$ & ${ }^{113} \mathrm{Cd}$ & 0.5800 \\
${ }^{133} \mathbf{C s}$ & 55 & 78 & 2.058 & $(n, \gamma)$ & ${ }^{134} \mathrm{Cs}$ & 0.6620 \\
${ }^{151} \mathbf{E u}$ & 63 & 88 & 1.822 & $(n, \gamma)$ & ${ }^{152} \mathrm{Eu}$ & 1.8180 \\
${ }^{154} \mathbf{S m}$ & 62 & 92 & 0.253 & $(n, \gamma)$ & ${ }^{155} \mathrm{Eu}$ & 0.1470 \\
${ }^{226} \mathbf{R a}$ & 88 & 138 & 0.044 & $(n, \gamma)$ & ${ }^{227} \mathrm{Ac}$ & 0.0448 \\
\hline
\end{tabular}

\subsection{The nuclear densities}

The ".nuclear density" is the .most .useful .observation for analyzing the nuclear structure that .may be constructed from the eigen-functions of an .adjustable singleparticle potential of Harmonic Oscillator (HO) type. It gives as much detailed information on the "internal structure" of nuclei, since they are "directly related" to the wave function of the "nucleons". In a general point of view, the calculated nuclear density profiles reveal that the density at the center of an atomic nucleus decreases slightly with increasing mass. This implies that, as the mass number of each nucleus increases, the central density undergoes a slight diffusion, while it becomes less dense with increasing radius and volume [18]. The charge density distributions were derived by folding a proton's charge distributions with the proton density calculated using the HO wave functions, as in the equation below:

$$
R_{r m c}^{c}=\left[\left(R_{r m c}^{p}\right)^{2}+(0.8)^{2}\right]^{1 / 2} f m
$$

where $0.8 \mathrm{fm}$ is the rms charge radius of the proton [19].

In computing the observable charge densities using SHF method, another fact should also be considered, which is that the nucleons themselves have intrinsic electromagnetic structure. Thus, one has to compute the proton, neutron, and mass densities of the nucleons. To illustrate the behavior of the one particle nuclear density distribution, the 
calculated charge, proton, neutron and mass densities of the target nuclei are shown in Figure-1. Different colors are used to differentiate between the different nuclear densities of the nucleons .

From the resulting plots, we can deduce that, in the case of heavy nuclei, the nuclear density is constant near the center and falls fairly rapidly to zero at the nuclear surface. Their neighbors, so that, when one adds a new particle to the system, most of the others are not directly affected by its presence. Thus, the interior density does not substantially changed by increasing the number of the value of $\rho_{0}$, which is in the range of $0.05-0.18 \mathrm{fm}^{-3}$ for medium to heavy nuclei and decreases slowly with increasing mass number.

From these Figures, it can be seen that the central nuclear densities gradually decrease as the nucleons number (A) increases, which is due to the change in the self-consistent HF potential resulting from the additional nucleons. This change in charge density is available in the interior and the surface regions of the nuclei. The contribution of additional nucleons to the density is directly associated to the orbits that are filled. These orbits are changing the densities in the interior and the surface regions.
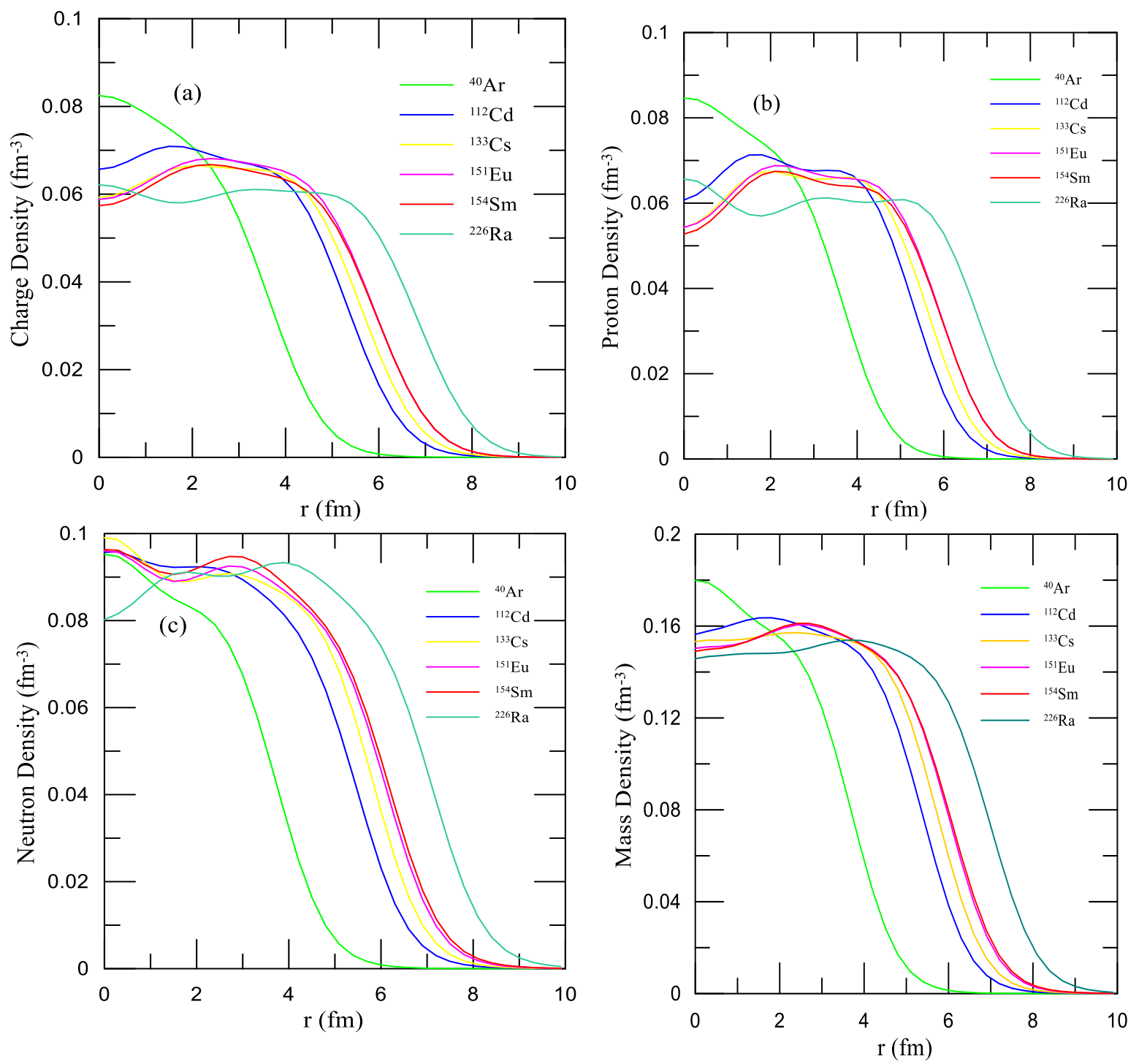

Figure 1-The calculated (a) charge, (b) proton, (c) neutron and (d) mass densities of the selected target nuclei using $\mathrm{SkM}^{*}$ parameterization. 


\subsection{Root Mean Square Radii and Neutron Skin Thickness}

The calculated charge, proton, neutron and mass rms radii using $\mathrm{SkM}^{*}$ parameterization are tabulated in Table-3 and depicted in Figure- 2, in comparison with experimental .charge .rms values taken from an earlier work [20].

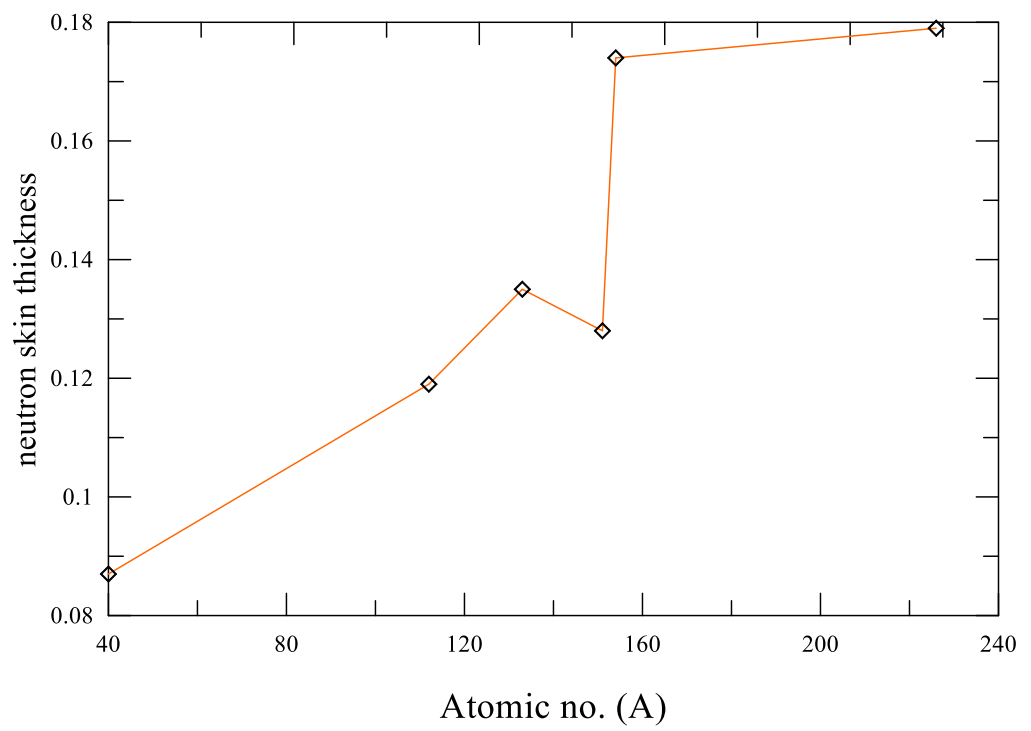

Figure 3-The neutron skin thickness for ${ }^{40} \mathrm{Ar},{ }^{112} \mathrm{Cd},{ }^{133} \mathrm{Cs},{ }^{151} \mathrm{Eu},{ }^{154} \mathrm{Sm}$, and ${ }^{226} \mathrm{Ra}$ elements using $\mathrm{SkM}^{*}$ Skyrme parameterization.

Table 3-The calculated charge, neutron, proton and mass rms radii using SkM* Skyrme parameterization in comparison with experimental charge rms taken from Ref. [20]

\begin{tabular}{ccccccc}
$\begin{array}{c}\text { Target } \\
\text { Nuclide }\end{array}$ & Mass & Neutron & Proton & $\begin{array}{c}\text { Charge } \\
\text { Theo. }\end{array}$ & $\begin{array}{c}\text { Charge } \\
\text { Exp.[20] }\end{array}$ & $\mathbf{t}_{\mathbf{n}}=\mathbf{r}_{\mathbf{n}}-\mathbf{r}_{\mathbf{p}}$ \\
\hline${ }^{\mathbf{4 0}} \mathbf{A r}$ & 3.393 & 3.432 & 3.345 & 3.436 & 3.427 & 0.087 \\
${ }^{112} \mathbf{C d}$ & 4.578 & 4.628 & 4.509 & 4.581 & 4.594 & 0.119 \\
${ }^{133} \mathbf{C s}$ & 4.830 & 4.885 & 4.750 & 4.813 & 4.804 & 0.135 \\
${ }^{151} \mathbf{E u}$ & 5.031 & 5.084 & 4.956 & 5.010 & 5.052 & 0.128 \\
${ }^{\mathbf{1 5 4}} \mathbf{S m}$ & 5.074 & 5.143 & 4.969 & 5.021 & - & 0.174 \\
${ }^{\mathbf{2 2 6}} \mathbf{R a}$ & 5.743 & 5.812 & 5.633 & 5.682 & 5.721 & 0.179 \\
\hline
\end{tabular}

It was found that the calculated rms radii increased with increasing the mass number. The theoretical charge rms radii increased from 3.436 for ${ }^{40} \mathrm{Ar}$ to 5.682 for ${ }^{226} \mathrm{Ra}$ and have a good agreement with experimental data.

To determine neutron thicknesses, based on Eq. 17, the rms neutron and proton density radii were exploited in Table-3, where the calculated neutron skin thickness $t$. The neutron thicknesses for the ${ }^{40} \mathrm{Ar},{ }^{112} \mathrm{Cd},{ }^{133} \mathrm{Cs},{ }^{151} \mathrm{Eu},{ }^{154} \mathrm{Sm}$, and ${ }^{226} \mathrm{Ra}$ nuclei are given in the last column of Table-3 and displayed in Figure-3. The theoretical $t_{n}$ values were increased with increasing mass number.

\subsection{Binding Energies}

The obtained binding energies of the selected target nuclei $\left({ }^{40} \mathrm{Ar},{ }^{112} \mathrm{Cd},{ }^{133} \mathrm{Cs},{ }^{151} \mathrm{Eu},{ }^{154} \mathrm{Sm}\right.$, and ${ }^{226} \mathrm{Ra}$ nuclei) are given in Table-4, in comparison with the corresponding experimental data taken from Ref. [21]. The results .are also displayed graphically in Figure-4. Both the experimental and theoretical results of binding energies are dependent on mass number; when the mass number increases the binding energy increases. It is clear from Table 4 that all the calculations are in well agreement with the experimental data. 


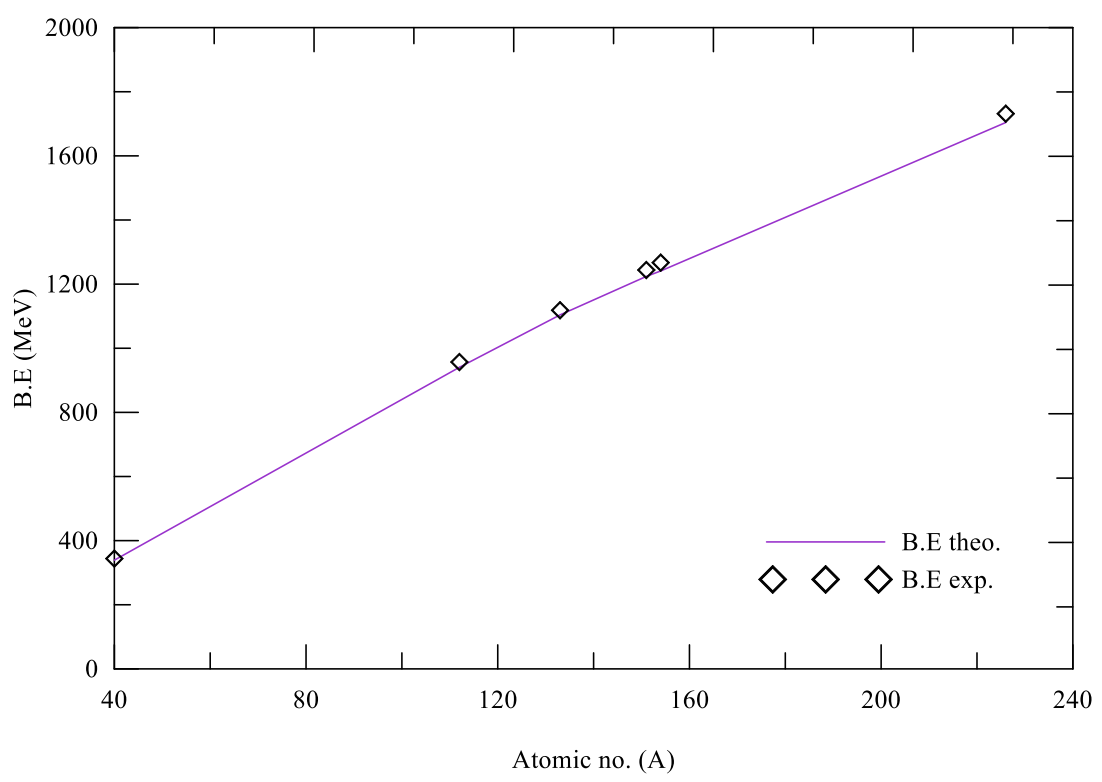

Figure 4-The binding energies for selected target nuclei $\left({ }^{40} \mathrm{Ar},{ }^{112} \mathrm{Cd},{ }^{133} \mathrm{Cs},{ }^{151} \mathrm{Eu},{ }^{154} \mathrm{Sm}\right.$, and ${ }^{226} \mathrm{Ra}$ nuclei) using $\mathrm{SkM}^{*}$ parameterization in comparison with the experimental data taken from Ref [21]

Table 4-The calculated total binding energies for selected target nuclei using $\mathrm{SkM}^{*}$ parameterization in comparison with the experimental data taken from Ref [21]

\begin{tabular}{rlr} 
Nuclide & B.E (MeV) & Exp [21] \\
\hline${ }^{\mathbf{4 0}} \mathbf{A r}$ & 339.03 & 343.816 \\
${ }^{112} \mathbf{C d}$ & 940.74 & $957.030 \pm 0.002$ \\
${ }^{133} \mathbf{C s}$ & 1104.20 & 1118.546 \\
${ }^{151} \mathbf{E u}$ & 1224.20 & $1244.161 \pm 0.002$ \\
${ }^{154} \mathbf{S m}$ & 1240.90 & $1266.961 \pm 0.003$ \\
${ }^{22} \mathbf{R a}$ & 1704.00 & $1731.635 \pm 0.002$ \\
\hline
\end{tabular}

\subsection{Cross-Sections for Bremsstrahlung Emission}

In this section, we discuss the emission of bremsstrahlung spectrum produced by total absorption of beta particles in the betavoltaic process.

In nuclear battery designs, the choice of a suitable radioisotope with a suitable energy of the ionization radiation is very important. It is dependent on the range of the energy emitted from the isotope in the material that the isotope is embedded in. For the betavoltaic process, a p-n junction in a semiconductor is used as a transducer. Beta particles transfer energy to the target material by Coulomb scattering and bremsstrahlung emission [22]. Since the incident beta particles have a mass that is equal to the mass of the target electrons, their paths of losing energy in a material will be random and a significant scattering will occur. Simulation of the beta particle tracks emitted from the pure beta emitters in silicon carbide $(\mathrm{SiC})$ semiconductor was investigated, and illustrated in Figure-6, using Monte Carlo code [23]. In this figure, the YZ section is where the beam is coming parallel to the $\mathrm{Z}$ axis and vertically down to the sample. The backscattering beta particles are shown in this figure with a red path. 


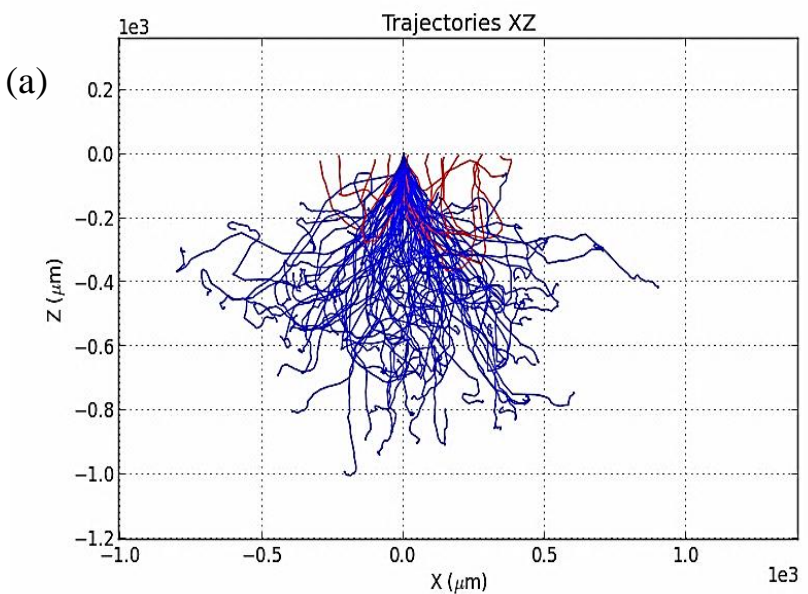

(c)

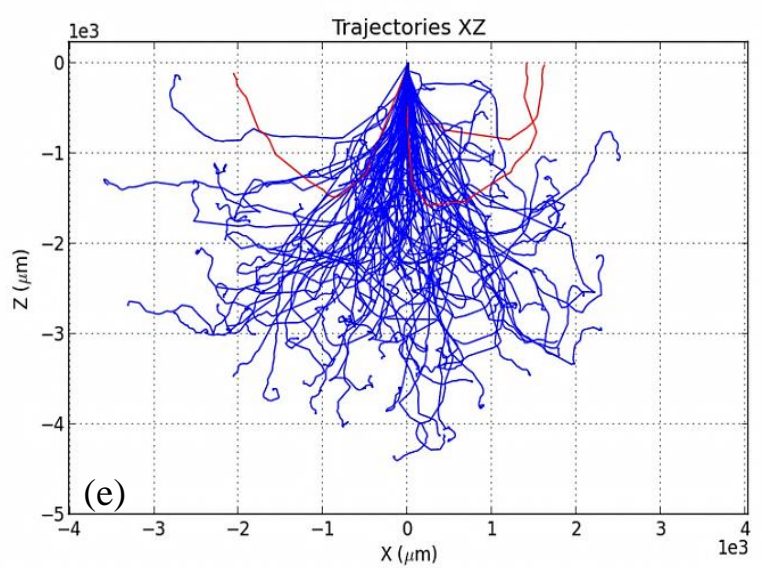

(E)

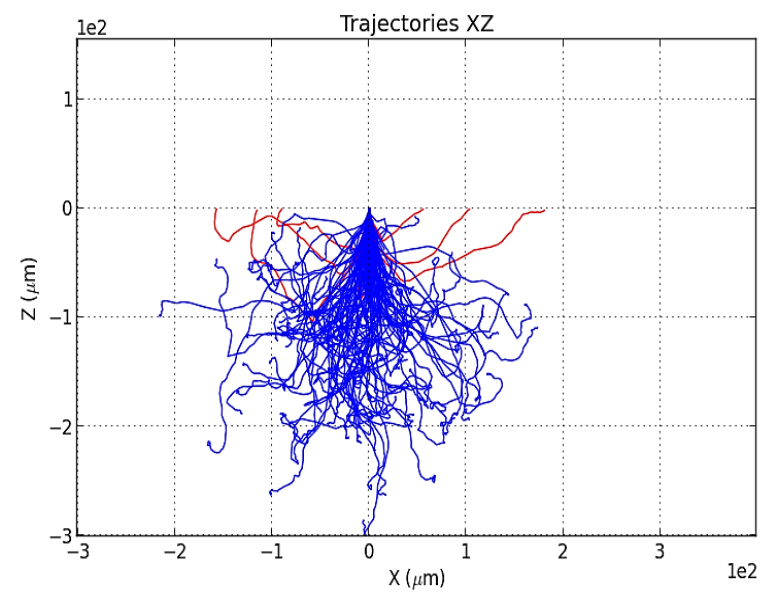

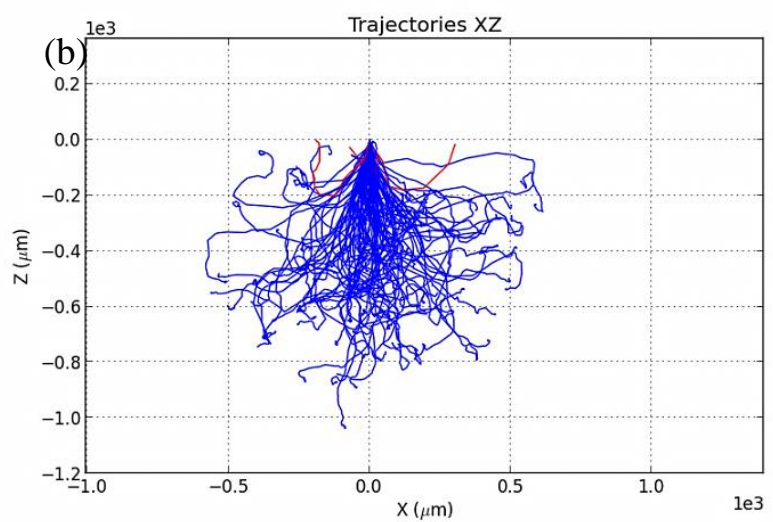

(d)

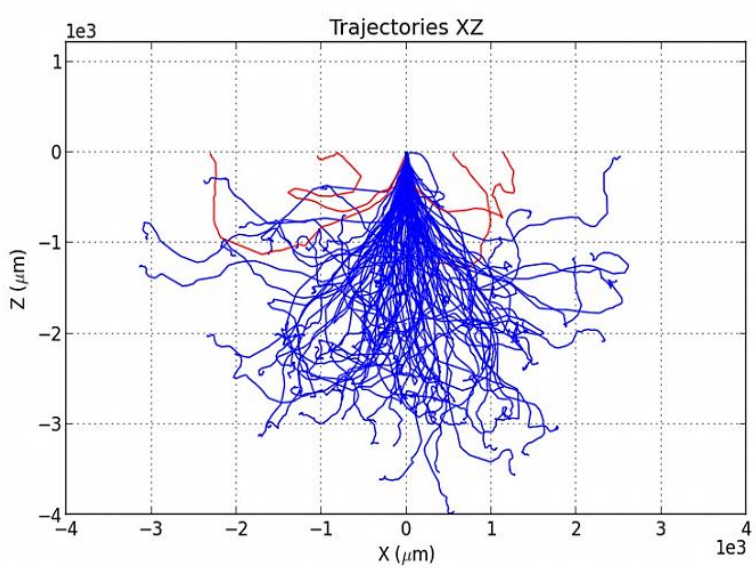

(F)

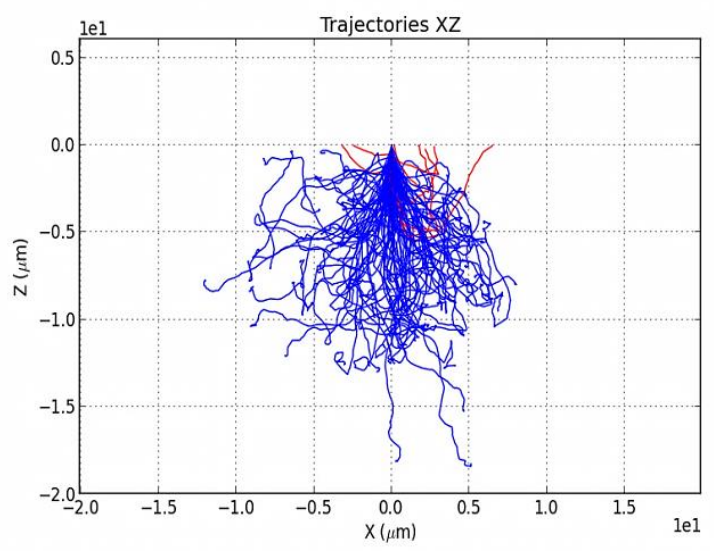

Figure 6-The trajectories of (a) ${ }^{42} \mathrm{Ar}(\mathrm{b}){ }^{113} \mathrm{Cd}$ (c) ${ }^{134} \mathrm{Cs}$ (d) ${ }^{152} \mathrm{Eu}$ (e) ${ }^{155} \mathrm{Eu}(\mathrm{f}){ }^{227} \mathrm{Ac}$ beta particles into silicon carbide with the beam entering a slab. The blue lines: absorbed within the sample, the red lines: backscattered (beta particle exits from the same surface as it entered). 
The generation of secondary gamma photons from bremsstrahlung events, produced by interaction of beta particles with the target nuclei, requires an additional shielding in battery design. Bremsstrahlung energy generation is effective when the transducer material has a high atomic number, since its scales approximately as $Z^{2}$ of the target. In the present work, the $\mathrm{SiC}$ semiconductor was used as a transducer material with relatively low atomic number. In Figure-7, we show the simulated bremsstrahlung spectra produced by beta particles impinging normally on the slab of SiC. It was found that the probability density of the generated bremsstrahlung decreases with increasing the bremsstrahlung energy. The comparison of the bremsstrahlung spectra with different energies are shown in Figure-8.
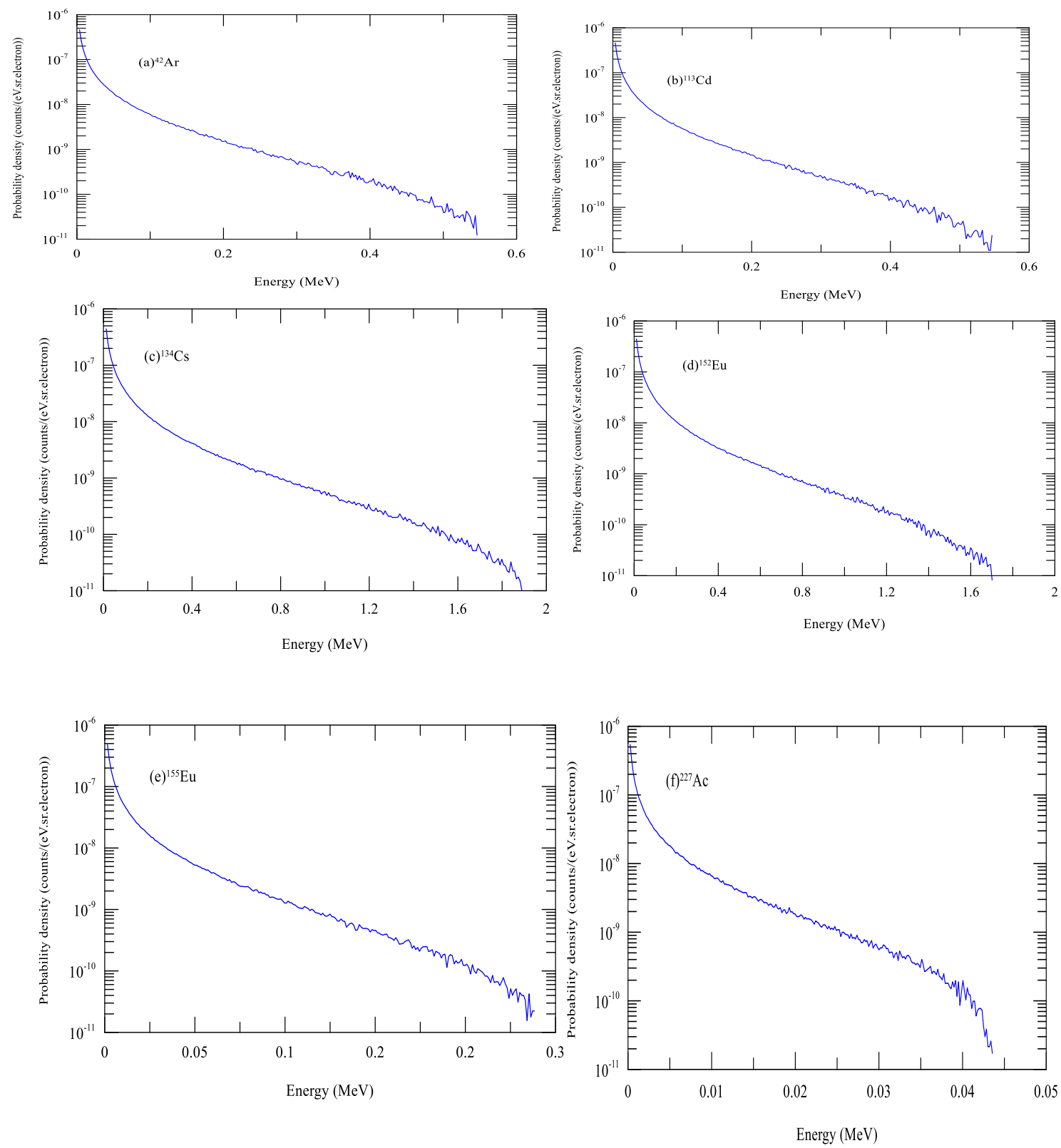

Figure 7-The bremsstrahlung spectrum produced by absorption of (a) ${ }^{42} \mathrm{Ar}(\mathrm{b}){ }^{113} \mathrm{Cd}(\mathrm{c}){ }^{134} \mathrm{Cs}(\mathrm{d}){ }^{152} \mathrm{Eu}$ (e) ${ }^{155} \mathrm{Eu}(\mathrm{f}){ }^{227} \mathrm{Ac}$ beta particles into silicon carbide. 


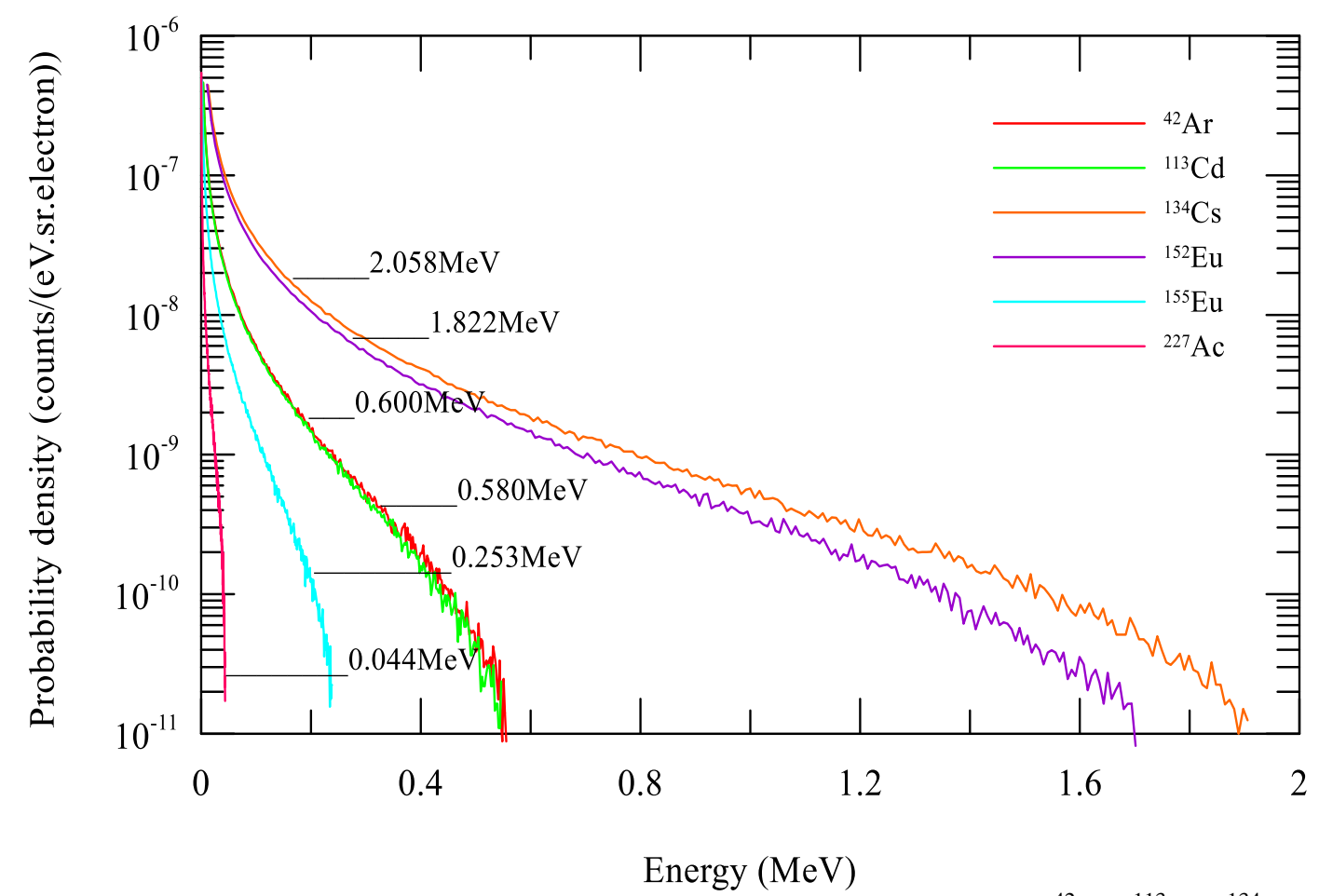

Figure 8-The bremsstrahlung spectra produced by absorption of target nuclei ${ }^{42} \mathrm{Ar},{ }^{113} \mathrm{Cd},{ }^{134} \mathrm{Cs},{ }^{152} \mathrm{Eu}$, ${ }^{155} \mathrm{Eu}$ and ${ }^{227} \mathrm{Ac}$ beta particles into silicon carbide.

\subsection{Backscattered Photons Specta}

In the present work, a Monte Carlo code was used to provide insight into gamma ray simulator results. Also, this section presents a backscattering probabilities simulation for the energy distributions, the backscattered probabilities of gamma photons of various energies, and multiple backscatters from the $\mathrm{SiC}$ semi-conductor used in nuclear batteries. As is usual for detailed Monte Carlo calculations, a gamma photon is represented by its energy, position coordinate $(\square, \varnothing)$, and direction cosines relative to some frame of reference. These quantities are chosen as randomly generated parameters, with the selection of the processes (ither photoelectric or Compton) when an interaction occurs. The code provides the energy distribution of backscattered photons after several scatterings in the semi-conductor.

The energy distribution of backscattered bremsstrahlung photons produced by absorption of beta particles emitted from ${ }^{42} \mathrm{Ar},{ }^{113} \mathrm{Cd},{ }^{134} \mathrm{Cs},{ }^{152} \mathrm{Eu},{ }^{155} \mathrm{Eu}$, and ${ }^{227} \mathrm{Ac}$ sources in $0.3 \mu \mathrm{m}$ thickness of $\mathrm{SiC}$ were calculated and depicted in Figure-9. For all sources, the energy distributions initially increases and reaches a maximum value. Thereafter, they decrease rapidly with increasing photons energy. Since the energy distributions occur in a small energy region, a semi-logarithmic graph is down. The backscattering energy distributions of gamma photons were calculated for different incident photon energies. The mechanism of interaction depends on the type of particle, energy, and density.

For more illustration, the backscattering energy distributions are totally shown in Figure-10 for all beta emitted sources. It was found that the backscattering spectrum has two clear peaks. It is considerable that the energy distributions of photons scattered backward from the various beta sources show peaks at approximately $0.00116-0.001672 \mathrm{MeV}$ in the selected energy range. This is caused by Compton scattering in the material. The second maximum of the backscattering energy distributions occurs at different energy values, compared to the other sources, probably due to the larger number of bremsstrahlung photons from higher energy beta source. The energy distributions of backscattering photons vary depending on the decrease in their probability of moving forward through the $\mathrm{SiC}$ thickness. The peak positions of the backscattered photons are shifted to higher energies as the incident photon energy increases. 

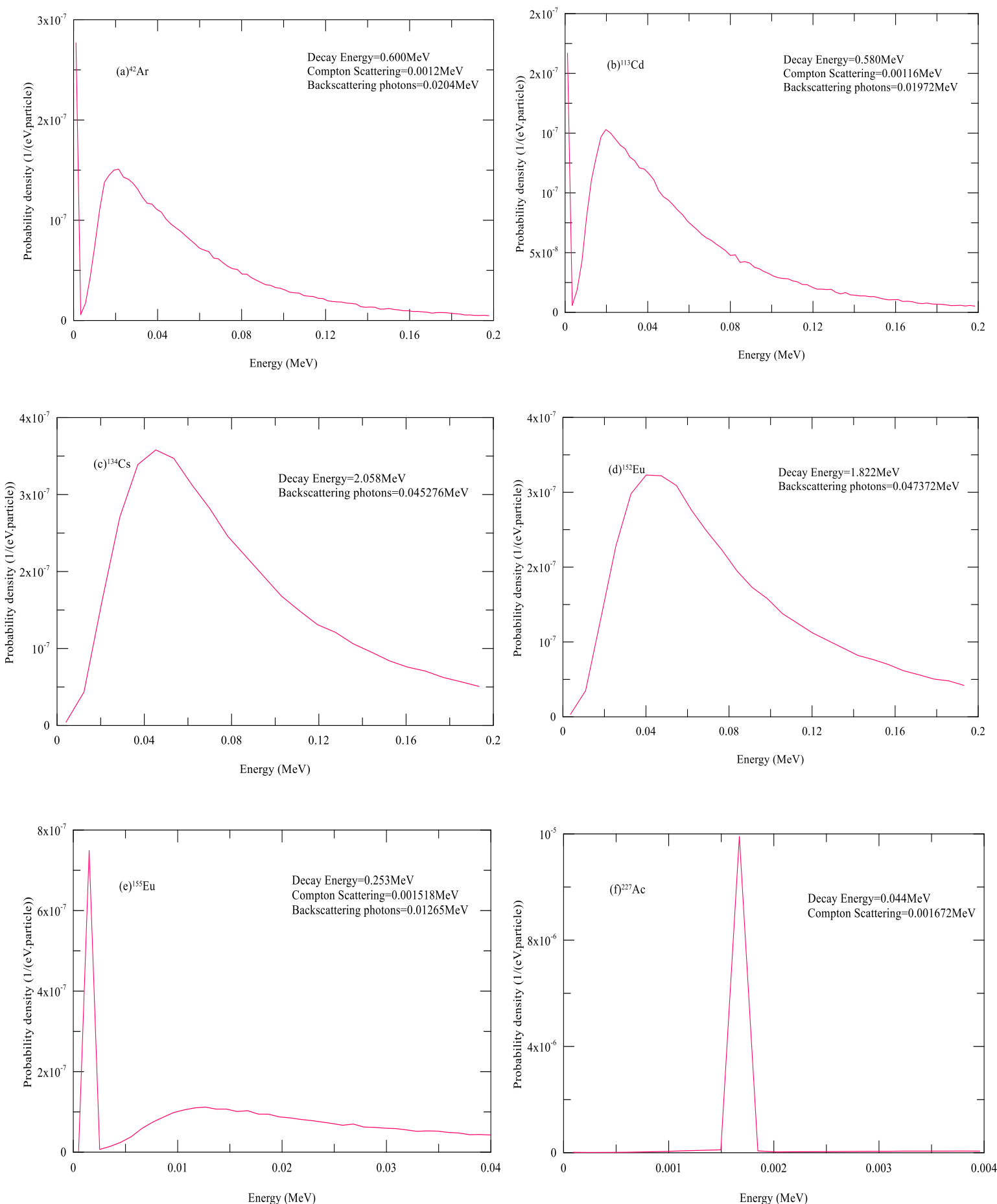

Figure 9- The backscattered bremsstrahlung photons produced by absorption of (a) ${ }^{42} \mathrm{Ar}$ (b) ${ }^{113} \mathrm{Cd}$ (c) ${ }^{134} \mathrm{Cs}(\mathrm{d}){ }^{152} \mathrm{Eu}(\mathrm{e}){ }^{155} \mathrm{Eu}(\mathrm{f}){ }^{227} \mathrm{Ac}$ beta particles into silicon carbide 


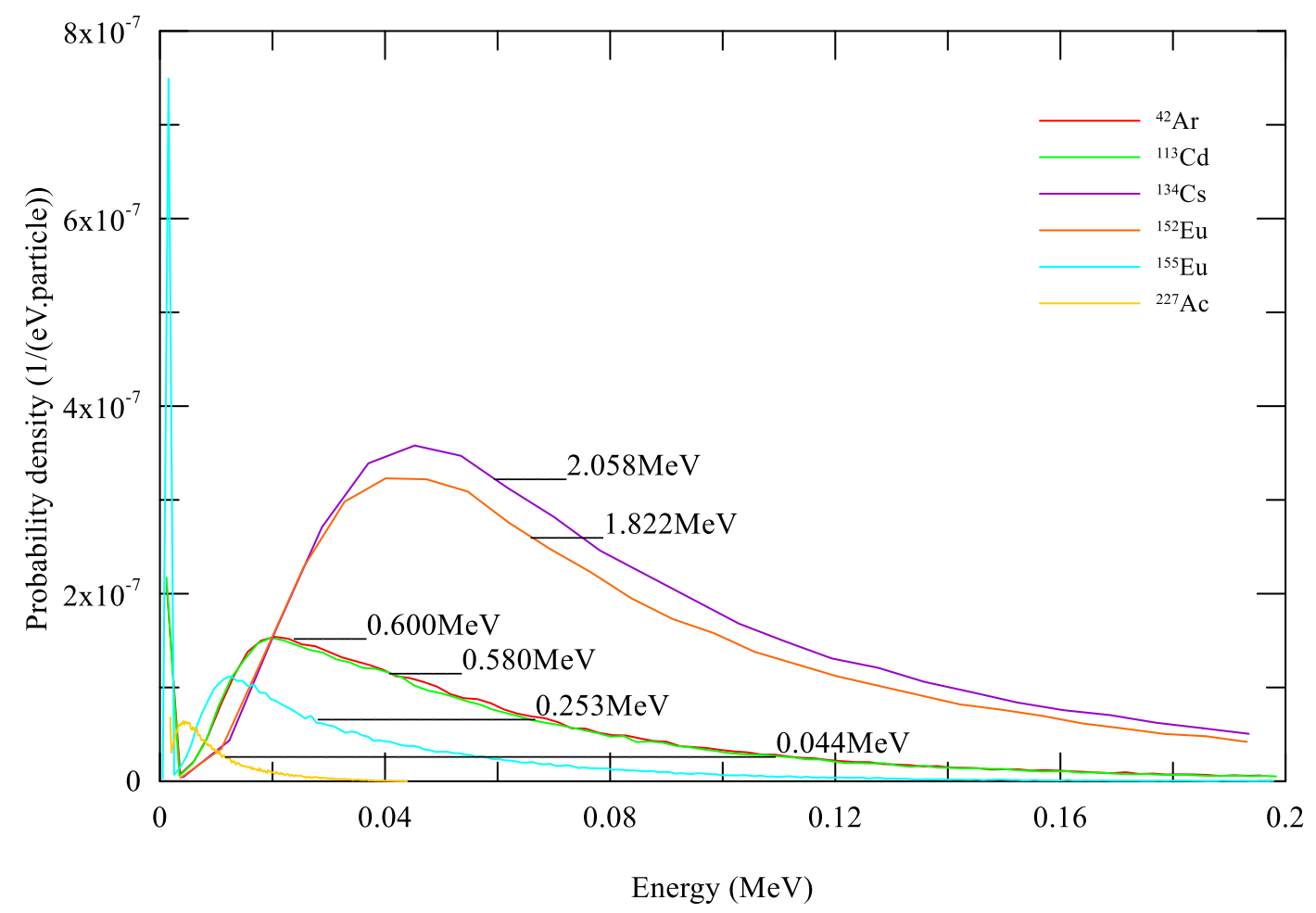

Figure 10-Comparison of the backscattered photon spectra produced by absorption of target nuclei $\left({ }^{42} \mathrm{Ar},{ }^{113} \mathrm{Cd},{ }^{134} \mathrm{Cs},{ }^{152} \mathrm{Eu},{ }^{155} \mathrm{Eu}\right.$, and $\left.{ }^{227} \mathrm{Ac}\right)$ beta particles into silicon carbide.

\section{Conclusions}

From the research performed here, it is possible to conclude that the binding energies and rms radii of the selected target nuclei, calculated using the SHF method with SkM* parameterization, are well reproduced and close to the experimental data. The SHF method is useful for calculating the spherical nuclei, because this force is central and has zero range interactions. The intensity of bremsstrahlung photons produced by beta particles absorption in $\mathrm{SiC}$ was investigated as a function of energy. It was observed that the intensity increases with increasing beta particle energies, while the backscattering photons also increase. This method can be used for the optimization of configuration of radiation protection in the fabrication of nuclear batteries. It also provides assistance to extend nuclear data used in nuclear battery technology and science.

\section{References}

1. Khajepoura and F. Rahmani. 2017. Appl. Radiat. Isot. 119(2017): 51.

2. Schmidt, G.R., Sutli, T.J. and Dudzinski, L.A. 2011. Radioisotope power: A key technology for deep space exploration in Radioisotopes Applications in Physical Sciences, ed. Nirmal Singh (2011), 419.

3. Kavetskiy et al., 2009. Appl. Radiat. Isot. 67(2009): 1057.

4. Qiao, D.Y., Chen, X.J., Ren, Y., Yuan, W.Z. 2011. J.of Microelectromechanical Systems (2011): 20.

5. Yakimov, E. B. 2016. Appl. Radiat. Isot. 112(2016): 98.

6. Vautherin,D. and Brink, D.M. 1972. Phys. Rev. C. 5 (1972) 626.

7. Decharge, J. and . Gogny, D. 1980. Phys. Rev. C. 21(1980): 1568.

8. Aytekin,H. Baldik, R. and Alici,H. 2012. Ann. Nucl. Enrgy, 46(2012): 128.

9. Chabanat, E. et al. 1997. Nucl. Phys. A. 627(1997): 710.

10. Reinhard, P. G. and Flocard,H. 1995. Nucl. Phys. A, 584(1995): 467.

11. Chabanat,E. Bonce,P. Haensel,P. Meyer, J. and Schaefer, R. 1998. Nucl. Phys. A, 635 (1998): 231. 
12. Reihard,P.G. 1991. Skyrme-Hartree-Fock calculation for Nuclear Ground State, in K. Langanke, J. A. Maruhn and S.E. Koonin "Computational Nuclear Physics I : Nuclear Structure, Spring Verlag Berlin 1 (1991): 28.

13. Alzubadi, 2015. Ind. J. Phys. 89(2015): 619.

14. Alzubadi, N. F. Latoofi and R. A. Radhi. 2015. Int. J. Mod. Phys. E, 24(2015): 1550099.

15. K.V.N. Sarma and K. Narasimha Murty. 1976. J. Phys. G. Nucl. Phys. 2(1976): 387.

16. M. Takehisa, T. Saito, T. Takahashi, Y. Sato, and T. Sato. 1993. Radiat. Phys. Chem. 42(1993): 495.

17. O. S. Uehara, S. Taniguchi, M. Kashiwagi, K. Mizusawa, and I. Sakamoto. 1993. Radiat. Phys. Chem. 42(1993): 515.

18. E. Tel, R. Baldik, H. Aytekin and A. Aydin. 2009. Anna. of Nucl. Energy, 36(2009): 1333.

19. E. Chabanat P. Bonce, P. Haensel, J. Meyer and R. Schaefer.1997. Nucl. Phys. A. 627(1997): 710.

20. Angeli and K.P. Marinova. 2013. J. Atom. Nucl. Dat. Table, 99(2013).

21. http://cdfe.sinp.msu.ru/services/

22. M.A. Prelas, C.L. Weaver, M.L. Watermann, E.D. Lukosi, R.J. Schott, and D.A.Wisniewski.2014. Nucl.Energy, 75(2014): 117.

23. D. W. O. Rogers, B. A. Faddegon, G. X. Ding, C.M. Ma, J. We, and T. R. Mackie. 1995. Medical phys. 22, 5(1995): 503. 